\title{
Teaching NeuroImages: High-resolution MRI before and during a sentinel headache demonstrates aneurysm wall hemorrhage
}

Radhia Ait Chalal, MD, Myriam Edjlali, PhD, Wagih Ben Hassen, MD, Catherine Lamy, MD, Gregoire Boulouis, MD, Christine Rodriguez Regent, MD, Denis Trystram, MD, Jean-Francois Meder, PhD, Catherine Oppenheim, PhD, and Olivier Naggara, PhD

Neurology ${ }^{\circledR}$ 2020;95:e224-e225. doi:10.1212/WNL.0000000000009774

A $3 \mathrm{~T}$ brain MRI, performed in a 48-year-old woman presenting with progressive headaches, demonstrated a 20-mm unruptured saccular basilar artery aneurysm. High-resolution vesselwall MRI showed a chronic mural thrombus and a circumferential aneurysm wall enhancement (figure), an imaging marker of aneurysm instability. ${ }^{1}$ Two days later, she had

\author{
Correspondence \\ Dr. Naggara \\ o.naggara@ghu-paris.fr
}

\section{MORE ONLINE}

$\rightarrow$ Teaching slides

links.lww.com/WNL/

B120

Figure High-resolution vessel wall MRI before and during sentinel headache

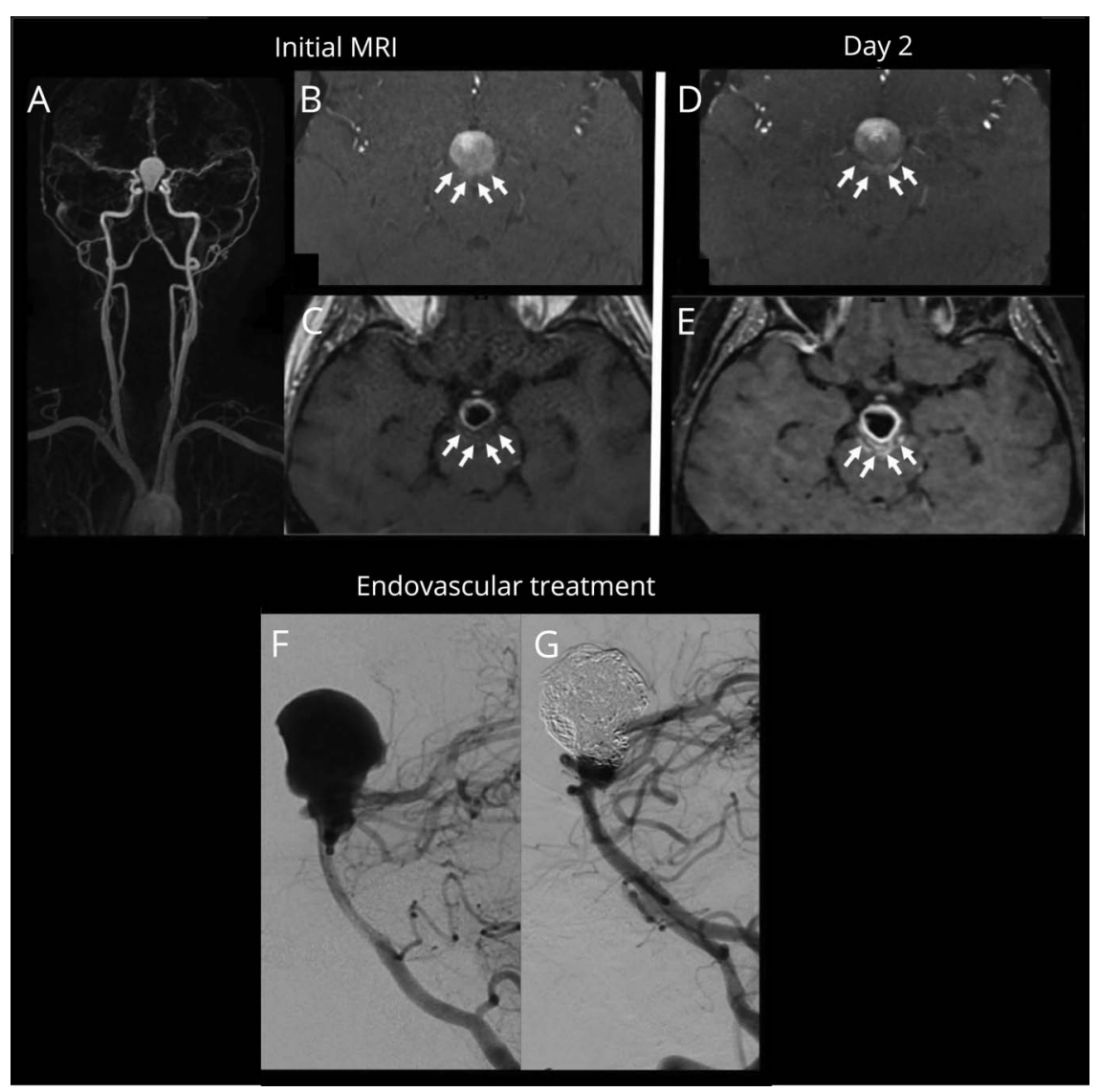

(A) Giant basilar tip aneurysm on magnetic resonance angiography. Comparison between high-resolution vessel-wall imaging performed before $(B, C)$ and during sentinel headache $(D, E)$ demonstrates aneurysm wall mural hematoma modification with $\mathrm{T} 1$-crescentic hyperintensity and contrast uptake (arrows) and thickened circumferential aneurysm wall enhancement. Digital subtracted angiography, lateral view, is shown before (F) and after (G) aneurysm coiling.

From the Departments of Neuroradiology (R.A.C., M.E., W.B.H., G.B., C.R.R., D.T., J.-F.M., C.O., O.N.) and Neurology (C.L.), Centre Hospitalier Sainte-Anne, GHU Paris Psychiatrie \& Neurosciences, Paris University, INSERM UMR 1266, Paris, France.

Go to Neurology.org/N for full disclosures. Funding information and disclosures deemed relevant by the authors, if any, are provided at the end of the article. 
a transient thunderclap headache, considered the worst headache she had ever reported. Repeated 3T MRI demonstrated acute mural hemorrhage without subarachnoid hemorrhage. Sentinel headaches, reported in every fourth patient preceding aneurysm rupture, ${ }^{2}$ have been interpreted as reflecting a warning for subarachnoid leak or, alternatively, structural wall changes, such as stretching or acute mural hemorrhage.

\section{Study funding}

No targeted funding reported.

\section{Disclosure}

The authors report no disclosures relevant to the manuscript. Go to Neurology.org/N for full disclosures.

\section{Appendix Authors}

\begin{tabular}{|c|c|c|}
\hline Name & Location & Contribution \\
\hline $\begin{array}{l}\text { Radhia Ait } \\
\text { Chalal, MD }\end{array}$ & $\begin{array}{l}\text { Department of } \\
\text { Neuroradiology, Centre } \\
\text { Hospitalier Sainte-Anne, } \\
\text { GHU Paris Psychiatrie \& } \\
\text { Neurosciences, Paris } \\
\text { University, INSERM UMR } \\
\text { 1266, Paris, France }\end{array}$ & $\begin{array}{l}\text { Analysis and } \\
\text { interpretation of data }\end{array}$ \\
\hline $\begin{array}{l}\text { Myriam } \\
\text { Edjlali, PhD }\end{array}$ & $\begin{array}{l}\text { Department of } \\
\text { Neuroradiology, Centre } \\
\text { Hospitalier Sainte-Anne, } \\
\text { GHU Paris Psychiatrie \& } \\
\text { Neurosciences, Paris } \\
\text { University, INSERM UMR } \\
\text { 1266, Paris, France }\end{array}$ & $\begin{array}{l}\text { Critical revision of } \\
\text { manuscript for } \\
\text { intellectual content, } \\
\text { analysis and } \\
\text { interpretation of data }\end{array}$ \\
\hline $\begin{array}{l}\text { Wagih Ben } \\
\text { Hassen, MD }\end{array}$ & $\begin{array}{l}\text { Department of } \\
\text { Neuroradiology, Centre } \\
\text { Hospitalier Sainte-Anne, } \\
\text { GHU Paris Psychiatrie \& } \\
\text { Neurosciences, Paris } \\
\text { University, INSERM UMR } \\
\text { 1266, Paris, France }\end{array}$ & $\begin{array}{l}\text { Critical revision of } \\
\text { manuscript for } \\
\text { intellectual content, } \\
\text { analysis and } \\
\text { interpretation of data }\end{array}$ \\
\hline $\begin{array}{l}\text { Catherine } \\
\text { Lamy, MD }\end{array}$ & $\begin{array}{l}\text { Department of Neurology, } \\
\text { Centre Hospitalier Sainte- } \\
\text { Anne, GHU Paris Psychiatrie } \\
\text { \& Neurosciences, Paris } \\
\text { University, INSERM UMR } \\
\text { 1266, Paris, France }\end{array}$ & $\begin{array}{l}\text { Analysis and } \\
\text { interpretation of data }\end{array}$ \\
\hline
\end{tabular}

Appendix (continued)

\begin{tabular}{|c|c|c|}
\hline Name & Location & Contribution \\
\hline $\begin{array}{l}\text { Gregoire } \\
\text { Boulouis, } \\
\text { MD }\end{array}$ & $\begin{array}{l}\text { Department of } \\
\text { Neuroradiology, Centre } \\
\text { Hospitalier Sainte-Anne, } \\
\text { GHU Paris Psychiatrie \& } \\
\text { Neurosciences, Paris } \\
\text { University, INSERM UMR } \\
\text { 1266, Paris, France }\end{array}$ & $\begin{array}{l}\text { Critical revision of } \\
\text { manuscript for } \\
\text { intellectual content, } \\
\text { analysis and } \\
\text { interpretation of data }\end{array}$ \\
\hline $\begin{array}{l}\text { Christine } \\
\text { Rodriguez } \\
\text { Regent, MD }\end{array}$ & $\begin{array}{l}\text { Department of } \\
\text { Neuroradiology, Centre } \\
\text { Hospitalier Sainte-Anne, } \\
\text { GHU Paris Psychiatrie \& } \\
\text { Neurosciences, Paris } \\
\text { University, INSERM UMR } \\
\text { 1266, Paris, France }\end{array}$ & $\begin{array}{l}\text { Analysis and } \\
\text { interpretation of data }\end{array}$ \\
\hline $\begin{array}{l}\text { Denis } \\
\text { Trystram, } \\
\text { MD }\end{array}$ & $\begin{array}{l}\text { Department of } \\
\text { Neuroradiology, Centre } \\
\text { Hospitalier Sainte-Anne, } \\
\text { GHU Paris Psychiatrie \& } \\
\text { Neurosciences, Paris } \\
\text { University, INSERM UMR } \\
\text { 1266, Paris, France }\end{array}$ & $\begin{array}{l}\text { Analysis and } \\
\text { interpretation of data }\end{array}$ \\
\hline $\begin{array}{l}\text { Jean- } \\
\text { Francois } \\
\text { Meder, PhD }\end{array}$ & $\begin{array}{l}\text { Department of } \\
\text { Neuroradiology, Centre } \\
\text { Hospitalier Sainte-Anne, } \\
\text { GHU Paris Psychiatrie \& } \\
\text { Neurosciences, Paris } \\
\text { University, INSERM UMR } \\
\text { 1266, Paris, France }\end{array}$ & $\begin{array}{l}\text { Critical revision of } \\
\text { manuscript for } \\
\text { intellectual content }\end{array}$ \\
\hline $\begin{array}{l}\text { Catherine } \\
\text { Oppenheim, } \\
\text { PhD }\end{array}$ & $\begin{array}{l}\text { Department of } \\
\text { Neuroradiology, Centre } \\
\text { Hospitalier Sainte-Anne, } \\
\text { GHU Paris Psychiatrie \& } \\
\text { Neurosciences, Paris } \\
\text { University, INSERM UMR } \\
\text { 1266, Paris, France }\end{array}$ & $\begin{array}{l}\text { Critical revision of } \\
\text { manuscript for } \\
\text { intellectual content }\end{array}$ \\
\hline $\begin{array}{l}\text { Olivier } \\
\text { Naggara, } \\
\text { PhD }\end{array}$ & $\begin{array}{l}\text { Department of } \\
\text { Neuroradiology, Centre } \\
\text { Hospitalier Sainte-Anne, } \\
\text { GHU Paris Psychiatrie \& } \\
\text { Neurosciences, Paris } \\
\text { University, INSERM UMR } \\
\text { 1266, Paris, France }\end{array}$ & $\begin{array}{l}\text { Study concept and } \\
\text { design, critical revision of } \\
\text { manuscript for } \\
\text { intellectual content, } \\
\text { study supervision }\end{array}$ \\
\hline
\end{tabular}

\section{References}

1. Edjlali M, Guédon A, Ben Hassen W, et al. Circumferential thick enhancement at vessel wall MRI has high specificity for intracranial aneurysm instability. Radiology 2018;289:181-187.

2. Beck J, Raabe A, Szelenyi A, et al. Sentinel headache and the risk of rebleeding after aneurysmal subarachnoid hemorrhage. Stroke 2006;37:2733-2737. 


\section{Neurology}

\section{Teaching NeuroImages: High-resolution MRI before and during a sentinel headache demonstrates aneurysm wall hemorrhage}

Radhia Ait Chalal, Myriam Edjlali, Wagih Ben Hassen, et al.

Neurology 2020;95;e224-e225 Published Online before print June 12, 2020

DOI 10.1212/WNL.0000000000009774

This information is current as of June 12, 2020

\section{Updated Information \&} Services

\section{References}

Subspecialty Collections

Permissions \& Licensing

Reprints including high resolution figures, can be found at: http://n.neurology.org/content/95/2/e224.full

This article cites 2 articles, 1 of which you can access for free at: http://n.neurology.org/content/95/2/e224.full\#ref-list-1

This article, along with others on similar topics, appears in the following collection(s):

MRI

http://n.neurology.org/cgi/collection/mri

Secondary headache disorders

http://n.neurology.org/cgi/collection/secondary_headache_disorders Stroke in young adults

http://n.neurology.org/cgi/collection/stroke_in_young_adults

Subarachnoid hemorrhage

http://n.neurology.org/cgi/collection/subarachnoid_hemorrhage

Information about reproducing this article in parts (figures,tables) or in its entirety can be found online at:

http://www.neurology.org/about/about_the_journal\#permissions

Information about ordering reprints can be found online:

http://n.neurology.org/subscribers/advertise

Neurology ${ }^{\circledR}$ is the official journal of the American Academy of Neurology. Published continuously since 1951, it is now a weekly with 48 issues per year. Copyright (C 2020 American Academy of Neurology. All rights reserved. Print ISSN: 0028-3878. Online ISSN: 1526-632X.

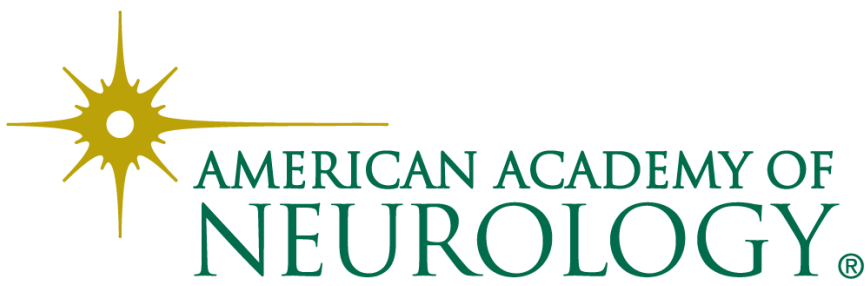

